nanoshell bioconjugates can be used to effectively target and image human epidermal growth factor receptor 2 (HER2-a relevant biomarker that is prevalent in human breast carcinoma cells). The researchers chose nanoshells composed of a dielectric silica core surrounded by a thin metallic shell composed of gold whose optical properties can be adjusted by controlling their dimensions. Nanoshells have a strong optical resonance that can be tuned in wavelength across the visible and infrared spectrum, allowing the relative contributions of scattering and absorption at a given wavelength to be tuned by controlling the dimensions of the core and the shell. In this study, nanoshells with a 240-nm-diameter silica core and a 35-nmthick gold shell were used. By utilizing these nanoshells, it is possible to conjugate bio-relevant materials, in this case, proteins that facilitate in vivo imaging.

The researchers attached a linker complex-either anti-HER2 or anti-IgG antibodies-PEG (poly ethylene glycol) - to the nanoshell surface. These conjugated nanoshells where exposed to HER2 expressing SKBr3 cells and studied by dark-field microscopy. A significant increase in optical contrast was observed in the HER2 positive cells targeted with anti-HER2-labeled nanoshells as compared to anti-IgG-labeled nanoshells or cells not exposed to the nanoshell conjugates. The researchers said that nanoshellbased conjugates offer the next generation to in vivo imaging due to their nearinfrared tunability, size flexibility, and systemic control of optical properties.

LARKEN E. EULISS

\section{Hybrid Photoelectrodes for Solar Water Splitting}

Efficient production of hydrogen from water using solar energy is a much-soughtafter research goal that has eluded scientists for many years. The wide-bandgap materials that are used in single-junction electrochemical devices to carry out water splitting processes typically absorb sunlight poorly, resulting in low solar-tohydrogen (STH) conversion efficiencies. Recently, E.L. Miller and colleagues from the Hawaii Natural Energy Institute at the University of Hawaii developed a prototype multijunction photoelectrode proposed to more effectively harness the sun's energy for hydrogen production than has been previously possible.

In the May issue of Electrochemical and Solid-State Letters (p. A247), the researchers reported on the performance characteristics of a prototype hybrid photoelectrode (HPE) device and discussed materials developments that will be nec- essary to improve the STH efficiency. An $\mathrm{HPE}$ is a monolithic thin-film device that integrates photoelectrochemical (PEC) and photovoltaic (PV) components in a multiple-junction arrangement. In such a device, the efficiency of hydrogen production is ideally proportional to the photocurrent in the PEC semiconductor, and thus optimum performance is achieved by maximizing this photocurrent.

The prototype HPE described and tested by the research group consists of an $a$ $\mathrm{SiGe} / a-\mathrm{SiGe}$ tandem structure coated with a sputtered $\mathrm{WO}_{3}$ thin film. This device operated at a hydrogen photocurrent level of $0.5 \mathrm{~mA} / \mathrm{cm}^{2}$ in outdoor sunlight conditions. This corresponds to an STH efficiency of $0.6 \%$. The researchers estimated that peak photocurrents of $0.7 \mathrm{~mA} / \mathrm{cm}^{2}$ could be obtained from this device.

Although the results are promising, these materials clearly do not offer optimal device performance; novel materials must be developed for use as the PEC and PV components. One upgrade suggested by the researchers is the use of "improved" sputtered $\mathrm{WO}_{3}$ coatings. These films offer peak photocurrents of $2.4 \mathrm{~mA} / \mathrm{cm}^{2}$, which represent a threefold increase in peak photocurrent and ideally a corresponding improvement in STH. The researchers predict that when integrated with a newly developed $a-\mathrm{Si} / a-\mathrm{Si}$ tandem structure, an HPE with this improved coating layer would yield STH efficiencies exceeding $2.2 \%$.

The chief limitation of HPE structures at the moment is the maximum photocurrent allowed by the top PEC semiconductor films. Doped $\mathrm{TiO}_{2}$ or $\mathrm{WO}_{3}$ films may offer a solution; these feature photocurrent values as high as $5 \mathrm{~mA} / \mathrm{cm}^{2}$. The researchers also suggest that appropriate combinations of PEC and PV materials may yield STH values in excess of $10 \%$. Clearly, much development is necessary to attain such efficient hydrogen production in HPE devices, but these prototype results point to possible significant improvements in efficiency.

\section{ANDY FRANCIS}

\section{Distribution of Nanoparticles in Photopolymer Controlled Holographically}

Applications for holograms include three-dimensional displays, data storage, and photonics. In recent years, holographic gratings have been fabricated from twocomponent mixtures of organic photopolymers and from polymer-dispersed liquid crystals. The incorporation of materials that extend the range of the refraction index profile will result in more efficient holograms. For example, a system com- posed of organic photopolymers and inorganic nanoparticles, which, unlike organic compounds, display a wide range of refractive indices $(n)$, were recently proposed by researchers from the Department of Electronics Engineering, University of Electro-Communications (UEC), Tokyo, although heretofore, the formation mechanism of such holographic gratings was not clearly understood.

As reported in the April 15 issue of Optics Letters, UEC researchers Y. Tomita and N. Suzuki and K. Chikama from Chemical Research Laboratories, Nissan Chemical Industries, demonstrated holographic control of morphology in nanoparticle-dispersed photopolymers and explained the formation of the holographic gratings in relatively simple terms of the chemical potential $(\mu)$ of non-interacting particles. The researchers combined either $\mathrm{SiO}_{2}$ nanoparticles $(n=1.46$, diameter $=$ $13 \mathrm{~nm})$ or $\mathrm{TiO}_{2}$ nanoparticles $(n=2.55$, diameter $=15 \mathrm{~nm}$ ) at a volume fraction of 0.34 with methacrylate monomers $(n=1.55$ in the liquid and 1.59 in the solid phase at a wavelength of $589 \mathrm{~nm}$ ) and the initiator titanocene to cast films $\sim 50 \mu \mathrm{m}$ thick. Transmission-type holograms were then recorded at a grating spacing of $1 \mu \mathrm{m}$ by exposing the films to two mutually coherent beams at a wavelength of $532 \mathrm{~nm}$. The researchers used transmission electron microscopy to show that the nanoparticles followed the intensity interference fringe pattern at a grating spacing of $1 \mu \mathrm{m}$.

Tomita and his colleagues hypothesized a mutual diffusion model in which monomers polymerize in the bright regions, where their $\mu$ decreases, leading to the diffusion of monomers from dark to bright regions. The photoinsensitive inorganic nanoparticles, on the other hand, are not consumed during polymerization, and their $\mu$ increases in the bright regions, causing them to diffuse to the dark regions. The researchers reasoned that the phase shift $(\phi)$ between the intensity interference fringe pattern and the recorded holograms should be $0^{\circ}$ for the $\mathrm{SiO}_{2}$ hologram, that is, the change in $n(\Delta n)$ is highest in the bright regions, because $n$ for $\mathrm{SiO}_{2}$ is less than $n$ for the polymer. Similarly, $\phi$ for the $\mathrm{TiO}_{2}$ hologram should be $180^{\circ}$, that is, $\Delta n$ is highest in the dark regions, because $n$ for $\mathrm{TiO}_{2}$ is greater than $n$ for the polymer. Both of these predictions were confirmed by the researchers.

Tomita and his colleagues said that their finding of all-optical control of nanoparticle distribution in photopolymers will facilitate the addition of new functionality to holographic applications, such as the fabrication of nonlinear periodic structures and nonlinear photonic 
crystals, through the incorporation of nonlinear optical nanoparticles. Other possibilities mentioned by the researchers include holograms with magnetic nanocrystals for magneto-optic applications and the incorporation of inorganic nanocrystals for multifunctional lightemitting devices.

STEVEN TROHALAKI

\section{Sequential Synthesis of Colloidal Type-II Core/Shell CdTe/CdSe Semiconductor Nanocrystals Demonstrated}

Semiconductor nanocrystals have been the subject of recent scientific and technological interest due to their promising potential applications, including photovoltaics and bio-imaging. In heterostructured nanocrystals, a higher-bandgap shell material can be grown onto a core material with a lower bandgap. Type II core-shell nanocrystals are materials engineered by their band offset $\left(\Delta E_{\mathrm{v}}\right)$, and in these structures, the band offsets are such that the energies of the conduction and valence bands of the shell are either both higher or both lower than those of the core. These materials can spatially separate and confine photo-generated holes and electrons. A common-cation system, CdTe (core)/CdSe (shell) nanocrystals are type II materials, with the majority of photogenerated holes confined in the CdTe core and the electrons in the CdSe shell. Recently, K. Yu and co-workers from the National Research Council of Canada have developed an efficient approach to the synthesis of high-quality CdTe/CdSe nanocrystals. For the CdSe shell, by control of various factors, the technique avoids the formation of a thicker CdSe nanocrystal layer resulting in a thinner shell, leading to a higher photolumines-

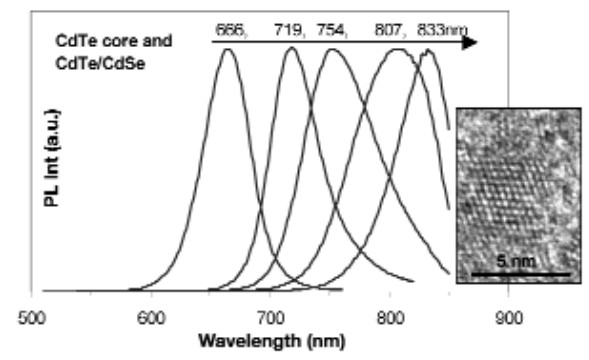

Figure 1. Photoemission spectra of $\mathrm{CdTe}$ (core)/ CdSe(shell) nanocrystals showing that the thicker the CoSe shell is, the more the emission redshifts. The inset is a transmission electron micrograph of one core-shell nanocrystal in an ensemble exhibiting $833 \mathrm{~nm}$ emission. cence efficiency. The researchers report their results in the March issue of Small.

Colloidal type II CdTe/CdSe nanocrystals were synthesized by the sequential addition of a tri-n-octylphosphine telluride (TOPTe)/tri- $n$-octylphosphine (TOP) solution and several shell-precursor solutions to a cadmium oxide (CdO)/TOP solution. The shell-precursor solutions consist of $\mathrm{CdO}$ and tri- $n$-octylphosphine selenide (TOPSe) in TOP. This synthetic approach is simple and does not involve either the addition of any acids, amines, or traditional tri-n-octylphosphine oxide (TOPO), or precipitation of core CdTe nanocrystals.

During the synthesis of the type-II $\mathrm{CdTe} / \mathrm{CdSe}$ nanocrystals, the researchers monitored the temporal evolution of the optical properties during the growth of the CdSe shell, as shown in the figure. The emission redshifts with increasing CdSe shell thickness.

This synthetic approach, similar to that of sequential anionic polymerization for well-defined block copolymers, is based on the knowledge gained during the search for synthetic routes for providing slow growth rates for high-quality CdSe and CdTe nanocrystals in large-scale production. It has been acknowledged that a slower particle growth rate results in less surface roughness, fewer surface defects, and higher photoluminescent efficiency. This synthetic approach is particularly well suited for realizing engineering materials with bandgaps in the nearinfrared and infrared spectral ranges.

GOPAL RAO

\section{Far-Field Technique for Visualization of Broadband Surface Plasmons Developed}

Surface plasmons (SPs) are traveling waves that propagate along the interface of a metal and an insulator. Commercially available sensors for environmental and biological applications have been fabricated by tailoring the interaction between impinging light and SPs. Recently, SPs have been explored as a means of realizing metallic photonic circuits. Imaging the SP intensity distribution is essential to characterizing SP-based devices. Traditionally, this imaging has been accomplished by near-field and fluorescence techniques. As reported in the April 15 issue of Optics Letters (p. 884), A. Bouhelier and G.P. Wiederrecht of the Chemistry Division and Center for Nanoscale Materials at Argonne National Laboratory have developed a far-field method for exciting and observing the SP intensity distribution. The SP intensity distribution was imaged by detecting leakage radiation, which are two of the four electromagnetic modes that are solutions to the dispersion relations.

The researchers deposited silver films onto glass substrates using thermal evaporation. To avoid total internal reflection of the radiative modes, an indexmatched, oil-immersed objective (numerical aperture of 1.4) was kept in contact with the glass substrate. The objective was part of an optical microscope, which was focused on the metal-glass interface. White light was used to excite a broadband surface plasmon continuum at the interface. This is possible because the objective introduced a distribution of wave vectors, through focusing, that fulfill the dispersion relation of the plasmon over the entire visible spectral region. The surface plasmon leakage radiation was recorded by a CCD camera that was placed in the image plane. A regeneratively amplified Ti:sapphire laser system was used to produce the incident whitelight continuum radiation, which was polarized by a multi-wavelength wave plate and a Glan-Thompson polarizer.

Propagating SPs were visualized by recording the real-space distribution of leakage radiation emitted by SP waves at the dielectric-metal interfaces. The images reveal a spatial variation in the spectral components of the SPs, which the researchers have termed "rainbow jets," that extend a significant distance along the direction of propagation of the SPs away from the incident continuum radiation spot. According to Bouhelier and Wiederrecht, the observation of surface plasmon leakage radiation gives a direct measurement of the SP propagation length and the damping mechanisms for each wavelength. The study of surface plasmons and light-SP interactions opens possibilities for photonics because they allow the concentration and propagation of light below the usual resolution limit, which may enable the realization of wavelength-sensitive optical devices.

JEREMIAH T. ABIADE

\section{Combination of Raman Scattering Techniques Achieve Single-Molecule Detection of Biomolecules}

As molecular biology advances, the detection of low quantities of biomolecules is proving critical for some applications, said researchers T.-W. Koo, S. Chan, and A.A. Berlin of the Precision Biology group at Intel Corporation in their article published in the May 1 issue of Optics Letters (p. 1024).

Surface-enhanced Raman scattering (SERS), which has been demonstrated as a single-molecule detection method, benefits from signal increases on the order of $10^{3}-10^{15}$ primarily because of the electro- 